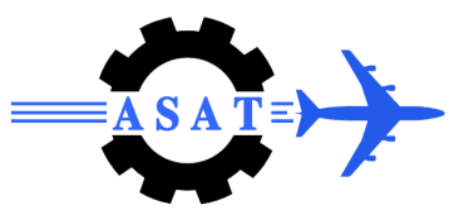

\title{
Tracking Controller Design for Nonlinear Dynamic Systems via Fuzzy Association Rule Reduction
}

\author{
G.A. Elnashar ${ }^{*}$
}

\begin{abstract}
One of the conventional significant criteria to be considered in real-time control applications is the computational complication of the controllers. The major weakness of fuzzy data mining is that after applying fuzzy data mining on the quantitative data, the number of extracted fuzzy association rules is very enormous. When many connection rules are obtained, the value of them will be reduced. This paper presents structures and a systematical progress method for two degree of freedom fuzzy controllers with nonhomogenous dynamics with respect to the two input channels. In this paper, singular value decomposition (SVD)-based complexity reduction technique and a reduced modified fuzzy logic controller (MFLC) are proposed. We introduce an approach to reduce and summarize the extracted fuzzy association rules after fuzzy data mining. Matlab/Simulink software is used to simulate the mathematical model of the Gun Turret-Barrel dynamic system. Different controllers are applied to the system. A comparison is introduced among the system performances under the control of each of FLC, a reduced FLC (in the number of membership functions and rules by using SVD method), and a reduced MFLC (reduction in the number of rules and modification in the shape of the membership functions by observing the system performance). The analysis points out that the proposed MFLC can ensure better control system performance with respect to the reference input in comparison with other controllers.
\end{abstract}

Keywords: Development of fuzzy controller, Fuzzy rule base reduction, Singular value decomposition (SVD), Tracking.

\section{Nomenclature}

\begin{tabular}{|c|c|c|c|}
\hline A & $\begin{array}{l}\text { the distance between the two motors in } \\
\text { the } \mathrm{x}_{1} \text { direction }\end{array}$ & M & The applied torque at motor \\
\hline A & Cross-section area of the arm. & $\mathrm{Q}_{\mathrm{i}}$ & $\begin{array}{l}\text { Generalized force corresponding to i- } \\
\text { coordinate. }\end{array}$ \\
\hline$c_{1}, \mathrm{~s}_{1}$ & $\cos \Theta_{1}$ and $\sin \Theta_{1}$ respectively & $\mathrm{T}$ & Total kinetic energy of the system. \\
\hline$c_{2}, \mathrm{~s}_{2}$ & $\cos \Theta_{2}$ and $\sin \Theta_{2}$ respectively & $\mathrm{V}$ & Total potential energy of the system. \\
\hline $\mathrm{D}$ & Dissipation function due to damping & $\rho$ & $\begin{array}{l}\text { The material density of the Gun Turret- } \\
\text { Barrel system. }\end{array}$ \\
\hline $\mathrm{L}$ & the arm length & $\Theta_{1}, \Theta_{2}$ & $\begin{array}{l}\text { angular position of motor " } 1 \text { " and motor " } 2 \text { " } \\
\text { respectively }\end{array}$ \\
\hline $\mathrm{m}_{\mathrm{p}}$ & Mass of the payload. & $\Theta$ & The actual position of the system. \\
\hline
\end{tabular}

\footnotetext{
*Egyptian Armed Forces, Egypt, enjygamal@ gmail.com 


\section{Introduction}

The fundamental problem of fire control is to orient a weapon so that the projectile it fires will hit the selected target. For weapons of the present era fire control varies in complexity from the simple aiming of a pistol to the intricate problem of destroying an intercontinental ballistic missile in flight. Two general methods of fire control are used with Army weapons: direct fire control and indirect fire control [1]. Direct fire control is used to control weapon fire delivered at a target that can be observed e.g., by optical or electro-optical instruments, either from the weapon itself or from nearby elements, i.e., as in a director-controlled type of weapon system. Indirect fire control is used for the control of weapon fire delivered at a target that cannot be observed from the weapon position. When the target is not directly visible from the weapon, e.g., when it lies behind a hill, an indirect method of observation is established. Fire control intelligence is then obtained and firing data computed for the gun at a fire direction center. In this paper, the mathematical model of the gun turret-barrel system will be derived. Dinavit_Hartinberg (DH) convention will be used to consider and rearrange the coordinate frames and to have the transformation matrices between any considered frames to the original frame. Also, Dynamic Modeling will be used to derive the Kinetic Energy, Potential Energy, and Virtual Work of each element in the system. Then, Lagrange's equation will be applied to have two equations of motion, the first is for the gun-turret and the second is for the gunbarrel.

Different types of controllers for controlling this system will be studied and discussed. Then the mathematical model of the system will be simulated under the control of different types of controllers. Comparisons between system performances under the control of each controller will be evaluated and discussed.

The paper is organized as follows. Section II presents the problem formulation and mathematical model of a Gun Turret-Barrel is derived. In Section III simulation is provided to demonstrate the design effectiveness. Finally, performance evolution and concluding remarks are made in Section $\mathrm{V}$

\section{Problem Formulation}

Automation and control of the gun turret-barrels is a need because of the ageing of the gun turret-barrel which works in the armed forces. This automation and control makes the task of the operators on the gun turret-barrel systems faster, easier, and more accurate as shown in Figure1. Automating of gun turret-barrels has many advantages. The first is to lessen the burden of the drive from the working group on the gun turret-barrel systems. Doing this leads to less time spent to track the targets associated to the gun turret-barrel (transient time). The second and the more important reason for automating the driving process is the increase of accuracy [2]. (minimizing the steady state error). The main objective of this paper is to develop a scheme for controlling a multi-body, multi-input, and multi-output nonlinear system. The system represented by a gun turret-barrel model consists of two subsystems: two motors driving two loads (turret for azimuth motion and barrel for elevation motion) coupled by nonlinear dynamics. In order to control the position (azimuth and elevation motions) of the gun turret-barrel, one must understand both the associated kinematics and dynamics. The equations of motion of the system to be controlled should be derived and verified.

To have the best tracking performance of the gun turret-barrel system, the derived equations of motion which include system's dynamics and kinematics should be simulated. The system's performance under the control of more than one control algorithm is evaluated. The selected controller scheme should present an acceptable tracking performance of the gun turret-barrel system under control. 


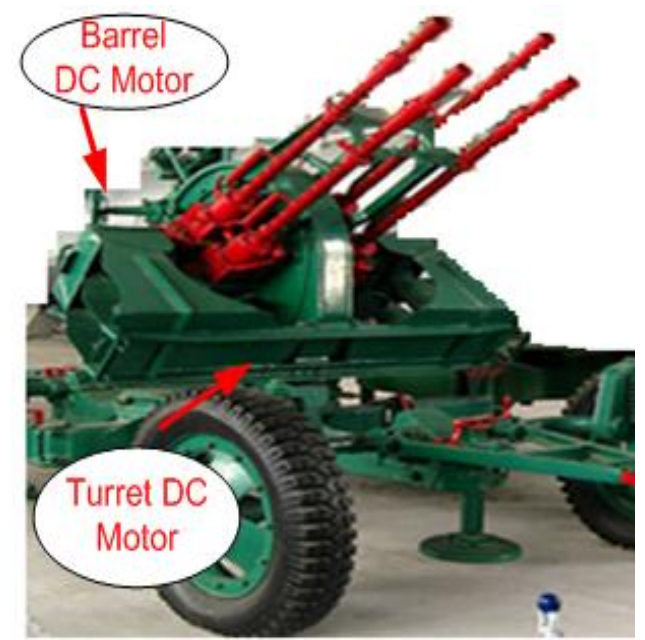

Fig. 1 Real Gun Turret-barrel system

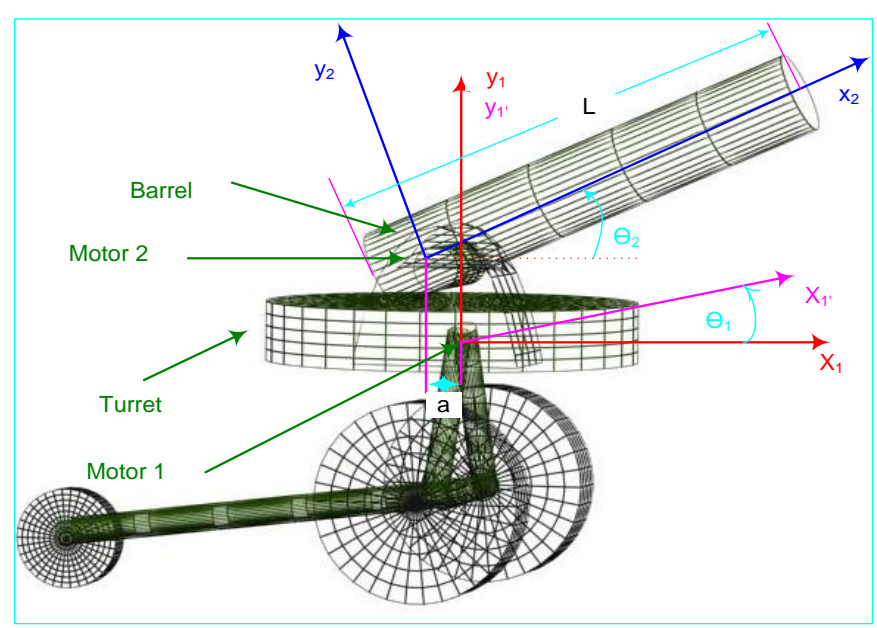

Fig. 2 A schematic diagram of the system

\subsection{Mathematical Model of a Gun Turret-Barrel}

The turn table shown in Figure 2, consists of two revolute joints (the first revolute joint is the Turret and the second is the Barrel) with two electric Direct Current (DC) motors, The Barrel of length " $L$ " is attached to the motor number " 2 ". A mathematical model of the Gun-Turret manipulator system including a dynamic model of a rigid has been studied. The dynamic modeling has been introduced using Lagrange's equation of motion and the direct Kinematics of the Gun-Barrel. The generalized coordinates have been chosen to describe the Turret motion and to apply Lagragian dynamic properly [2,3]. The generalized coordinate vector $\mathrm{q}$ is defined by $2 \times 1$ vector $q=\left[\begin{array}{ll}\theta 1 & \theta 2\end{array}\right] \mathrm{T}$. Hence, Lagrange's equations for the system are defined in general form as,

$$
\frac{d}{d t}\left(\frac{\partial T}{\partial \dot{q_{i}}}\right)-\frac{\partial T}{\partial q_{i}}+\frac{\partial V}{\partial q_{i}}+\frac{\partial D}{\partial \dot{q_{i}}}=Q_{i}
$$

Then, the total kinetic energy, potential energy, dissipation function (if we have damping) and the virtual work of the system must be obtained to apply Lagrangian dynamic modeling. Then, the equation of motion which describe the turntable motion is obtained by applying Lagrange's equation in the following form:

$$
\begin{gathered}
\frac{d}{d t}\left(\frac{\partial T}{\partial \dot{\Theta_{1}}}\right)-\frac{\partial T}{\partial \Theta_{1}}+\frac{\partial V}{\partial \Theta_{1}}=Q_{1} \\
{\left[\left(\frac{\rho A L^{3}}{3}+\mathrm{m}_{p} \mathrm{~L}^{2}\right) \mathrm{C}_{2}^{2}+\left(\rho \mathrm{AL}^{2} \mathrm{a}+2 \mathrm{~m}_{p} \mathrm{La}\right) \mathrm{C}_{2}\right.} \\
\left.+\left(\rho \mathrm{ALa}^{2}+\mathrm{I}_{R_{1}}+\mathrm{I}_{R_{2}}+\mathrm{m}_{R_{2}} \mathrm{a}^{2}+\mathrm{m}_{p} \mathrm{a}^{2}+\frac{1}{2} \mathrm{~m}_{1} \mathrm{R}_{1}^{2}\right)\right] \ddot{\Theta}_{1} \\
-\left[2\left(\frac{\rho A L^{3}}{3}+\mathrm{m}_{p} \mathrm{~L}^{2}\right) \mathrm{C}_{2} \mathrm{~S}_{2}+\left(\rho \mathrm{AL}^{2} \mathrm{a}+2 \mathrm{~m}_{p} \mathrm{~L} \mathrm{a}\right) \mathrm{S}_{2}\right] \dot{\Theta}_{2} \dot{\Theta}_{1}=\mathrm{M}_{1}
\end{gathered}
$$

For Arm's Equation of Motion, the second generalized coordinate $\mathrm{q}_{2}=\theta_{2}$ Lagrange's equation of motion can be applied as, 


$$
\frac{d}{d t}\left(\frac{\partial T}{\partial \dot{\Theta}_{2}}\right)-\frac{\partial T}{\partial \Theta_{2}}+\frac{\partial V}{\partial \Theta_{2}}=\mathrm{Q}_{2}
$$

Hence

$$
\begin{gathered}
\left(\frac{\rho A L^{3}}{3}+\mathrm{I}_{R_{2}}+\mathrm{m}_{\mathrm{P}} \mathrm{L}^{2}\right) \ddot{\Theta}_{2}+\left(\frac{\rho A L^{3}}{3} \mathrm{C}_{2} \mathrm{~S}_{2}+\mathrm{m}_{P} \mathrm{~L}^{2} \mathrm{C}_{2} \mathrm{~S}_{2}+\frac{\rho \mathrm{AL} \mathbf{L}^{2} a}{2} \mathrm{~S}_{2}+\right. \\
\left.\mathrm{m}_{P} \mathrm{LaS}_{2}\right) \ddot{\Theta}_{1}^{2}-9.81\left(\frac{\rho \mathrm{AL}}{2}+\mathrm{m}_{P} \mathrm{~L}\right) \mathrm{C}_{2}=\mathrm{M}_{2}
\end{gathered}
$$

The equations (2) and (3) describe the equation of motion of the system which consists of an arm based on a turntable. These equations can be rearranged in matrix form as:

$$
\begin{aligned}
& \left(\begin{array}{cc}
m_{11} & 0 \\
0 & m_{22}
\end{array}\right)\left(\begin{array}{c}
\ddot{\Theta}_{1} \\
\ddot{\Theta_{2}}
\end{array}\right)+\left(\begin{array}{cc}
D_{11} & 0 \\
0 & D_{22}
\end{array}\right) \dot{\Theta_{1}}\left(\begin{array}{c}
\dot{\Theta}_{1} \\
\dot{\Theta_{2}}
\end{array}\right)=\left(\begin{array}{l}
Q_{\Theta_{1}} \\
Q_{\Theta_{2}}
\end{array}\right) \\
& \text { where } \boldsymbol{m}_{\mathbf{1 1}}=\left(\frac{\rho \boldsymbol{A} \boldsymbol{L}^{3}}{\mathbf{3}}+\mathrm{m}_{P} \mathrm{~L}^{2}\right) \mathrm{C}_{\mathbf{2}}^{\mathbf{2}}+\left(\rho \mathbf{A} \mathbf{L}^{\mathbf{2}} \boldsymbol{a}+2 \mathrm{~m}_{P} \mathrm{La}\right) \mathrm{C}_{\mathbf{2}} \\
& +\left(\rho \mathrm{AL} a^{2}+\mathrm{I}_{R_{1}}+\mathrm{I}_{R_{2}}+\mathrm{m}_{R_{2}} a^{2}+\mathrm{m}_{p} a^{2}\right) \\
& m_{22}=\left(\frac{\rho A L^{3}}{3}+I_{R_{2}}+m_{p} L^{2}\right) \\
& D_{11}=-2\left(\frac{\rho A L^{3}}{3}+m_{P} L^{2}\right) C_{2} S_{2}-\left(\rho A L^{2} a+2 m_{P} L a\right) S_{2} \\
& \mathbf{D}_{22}=\left(\frac{\rho A L^{3}}{3} \mathbf{C}_{2} S_{2}+\mathbf{m}_{P} \mathbf{L}^{2} \mathbf{C}_{2} S_{2}+\frac{\rho \mathrm{AL}^{2} a}{2} \mathbf{S}_{2}+\mathbf{m}_{P} \operatorname{LaS}_{2}\right) \\
& Q_{\Theta_{1}}=M_{1}, \quad Q_{\Theta_{2}}=M_{2}+9.81\left(\frac{\rho \mathrm{AL}^{2}}{2}+m_{P} L\right) C_{2}
\end{aligned}
$$

The deduction from this mathematical model of the discussed Gun Turret-Barrel system is that each of the Turret and the Barrel has a nonlinear, multi-input-multi-output, and decoupled equation of motion. This inference leads to use a no conventional controller for controlling this Gun Turret-Barrel system.

\section{Simulation Results and Comparisons}

Mathematical simulation and analysis for the gun turret-barrel system is done by using the Matlab/Simulink software based on the derived mathematical model of the gun turret-barrel system in pervious section. As a case study parameter are defined as follows: Material density rho $=2000 \mathrm{Kg} / \mathrm{m} 3$, Barrel's length $\mathrm{L}=2 \mathrm{~m}$, Barrel's cross section $\mathrm{A}=0.1 \mathrm{~m}^{2}$, Mass of the payload $\mathrm{mp}=0.5 \mathrm{Kg}$, Mass of the rotor of the second motor $\mathrm{mR} 2=3 \mathrm{Kg}$, Mass moment of inertia of the rotor of the first and second motors are IR1 $=$ IR $2=0.5 \mathrm{Nm} / \mathrm{rad} / \mathrm{sec}^{2}$.

Different controller schemes will be used to control the system as follows: 
1. FLC with initial seven triangle membership functions for each input and output with 49 rules.

2. A modified FLC: a modification in the membership function shapes will be done to have a better performance.

3. A reduced modified FLC: a reduction in the number of rules will be done to minimize the memory required to implement the controller scheme.

4. A reduced modified FLC based on the SVD method: a modification in and reduction in the shape and the number of the used membership functions which also leads to a reduction in the number of the used rules.

\subsection{Fuzzy Logic Control}

To apply heuristic knowledge in the FLC, inputs, outputs, and universe of discourse are defined first. The inputs are the error $(\mathrm{E})$ between the reference position $\left(\theta_{\mathrm{r}}\right)$ and the actual position $\left(\theta_{\mathrm{a}}\right)$, and the change in error (CE). The output is the change in armature voltage (CU). The inputs and output illustrated in Figure (3) are described by: $\mathrm{E}=\mathrm{e}(\mathrm{k})=\Theta_{r}(\mathrm{k})-\Theta_{a}(\mathrm{k})$, $\mathrm{CE}=\mathrm{e}(\mathrm{k})-\mathrm{e}(\mathrm{k}-1), \mathrm{CU}=\mathrm{u}(\mathrm{k})-\mathrm{u}(\mathrm{k}-1)$, where $\mathrm{K}$ is the time index. The terms such as "Small" and "Big" are used to quantize the inputs and output values to linguistic terms that used to represent the input and output values are defined by seven fuzzy variables PB Positive Big, PM Positive Medium, PS Positive Small, ZE Zero, NS Negative Small, NM Negative Medium, and NB Negative Big. A FLC uses fuzzy rules to make a decision and generate the control effort. The rules are in the form of IF-THEN statements. For example, IF the error $(\mathrm{E})$ is equal to Positive Big (PB) and the change in error (CE) is equal to Positive Medium (PM) THEN the change in armature voltage $(\mathrm{CU})$ is equal to Negative Medium $(\mathrm{NM})[4,5]$.

The initial rules are constructed as shown in Table (1). The efficiency can be improved by adjusting the membership functions and rules.

To send out the armature voltage output, the output in the form of fuzzy sets must be converted to a crisp value. This process is called defuzzification. In this Paper, the center of gravity method is chosen.

A fuzzy rule base design has two important objectives. One is to achieve a good approximation. The other is to reduce the number of rules. The main difficulty is that these two objectives are contradictory.

\subsection{Singular Value Decomposition}

Complexity reduction is therefore becoming a pertinent research topic of fuzzy theory. SVD method is used to reduce the number of used rules by reducing the number of membership functions for each input and output. Doing this also modifies the shape of the membership functions $[6,7]$.

The first work is published in 1996 for rule bases using product-sum-gravity inference algorithm, piece-wise linear antecedents, and essentially, singleton consequents. Shortly after, the method is extended to using non-singleton consequents [8], Takagi-Sugeno type rule bases, and to a special fuzzy rule interpolation method. The method is further generalized in [9] to using SVD for reducing linguistic symbol arrays. A practical implementation technique is also proposed in for extremely large rule bases. An important advantage of the SVD reduction techniques is that there is a formal measure to filtering out the redundant and weakly contributing components. This implies that the degree of reduction can be applied according to the maximum acceptable error. For various cases, output error bound between the original set and the reduced set is readily expressible based on the sum of discarded singular values 

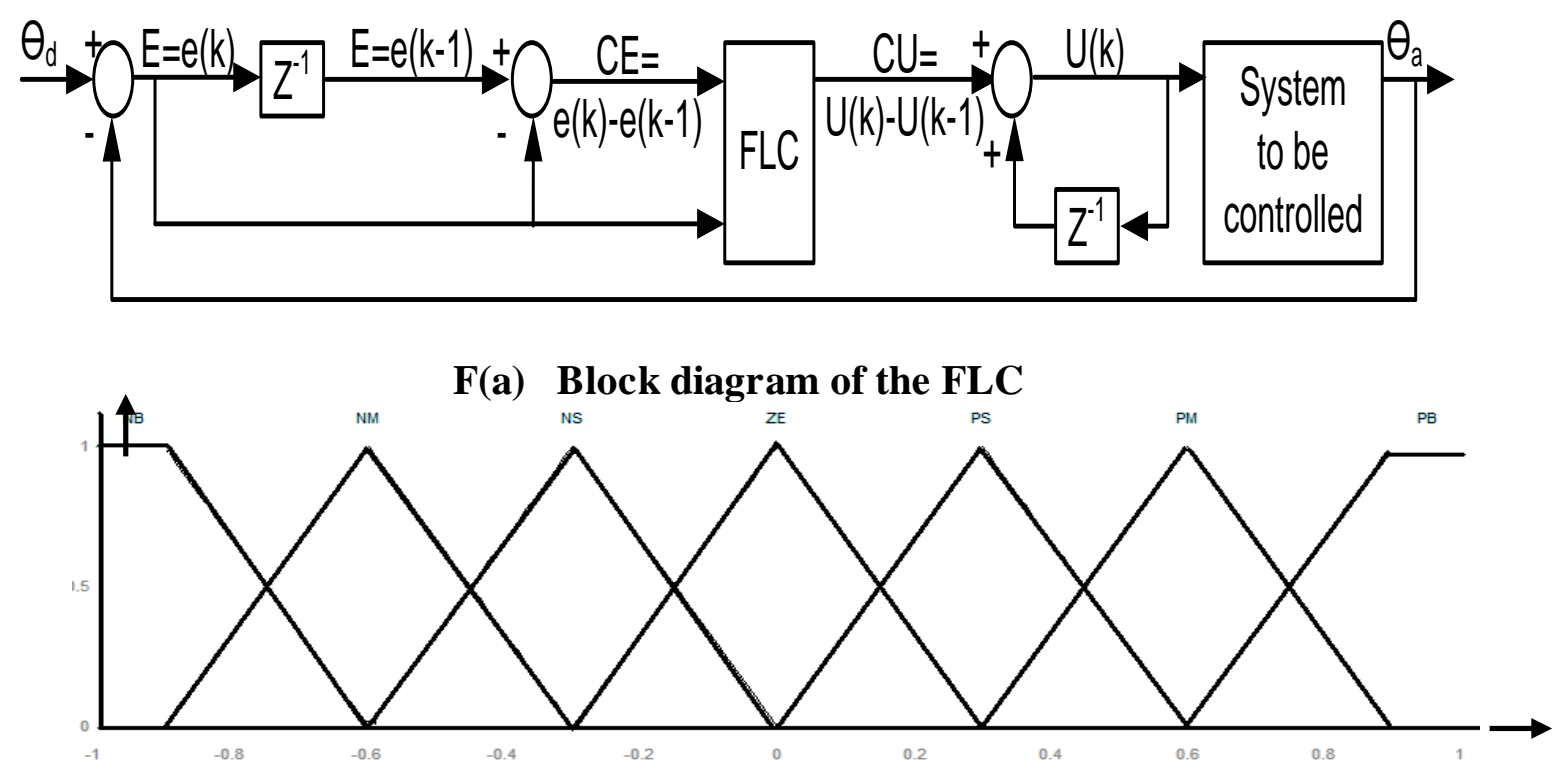

(b) Initial membership functions

Fig. 3 FLC Block diagram and initial membership output functions

Table 1 Initial Rules

\begin{tabular}{|c|c|c|c|c|c|c|c|}
\hline & NB & NM & NS & $\mathbf{Z E}$ & PS & $\mathbf{P M}$ & PB \\
\hline NB & NB & NB & NB & NB & $\overline{\mathrm{NM}}$ & NS & $\mathrm{ZE}$ \\
\hline NM & NB & NB & NB & $\mathrm{NM}$ & NS & $\mathrm{ZE}$ & PS \\
\hline NS & NB & NB & NM & NS & ZE & PS & $\mathrm{PM}$ \\
\hline$\overline{Z E}$ & NB & $\mathrm{NM}$ & NS & $\mathrm{ZE}$ & PS & PM & $\mathrm{PB}$ \\
\hline$\overline{\mathbf{P S}}$ & NM & NS & $\mathrm{ZE}$ & $\begin{array}{l}\text { PS } \\
\end{array}$ & $\mathrm{PM}$ & PB & $\mathrm{PB}$ \\
\hline PM & NS & ZE & PS & PM & PB & PB & $\mathrm{PB}$ \\
\hline PB & $\overline{Z Z E}$ & PS & PM & PB & PB & PB & PB \\
\hline
\end{tabular}

An M-file was made to represent and implement the SVD theory for membership functions reduction and modification. As will be shown in figure (4) the generated membership functions for the inputs are not a familiar one on the Simulink / Fuzzy toolbox, then it was a must to implement an M-file to reduce the number of rules and to modify the membership functions shapes on the base of the SVD method. The modified membership functions shapes which are generated by this M-file are shown in figure (4). Then, a comparison between system performances under the control of the initial FLC and the modified FLC by the SVD method are shown in figure (5) and table (2). Figure (5) shows the performance of the system under the control of each of a initial FLC, and the reduced one by the mean of SVD method.

In the previous case, a member ship function's modification and rule base reduction are done by observing the system's performance under the control of the initial FLC, these modification in the membership function and rule base reduction grantee that the system performance is not affected. But in this case study, the rule base reduction and membership function modification using the SVD [51] affects the performance (steady state error) of the system under the control of the controller derived by this method. But using the SVD method of [10] for the rule reduction has the advantage that it uses less number of rules compared with the used number of rules of the initial FLC. Table (2) shows the comparison between the system performance under the control of the initial FLC and the reduced one by the mean of the SVD method. 


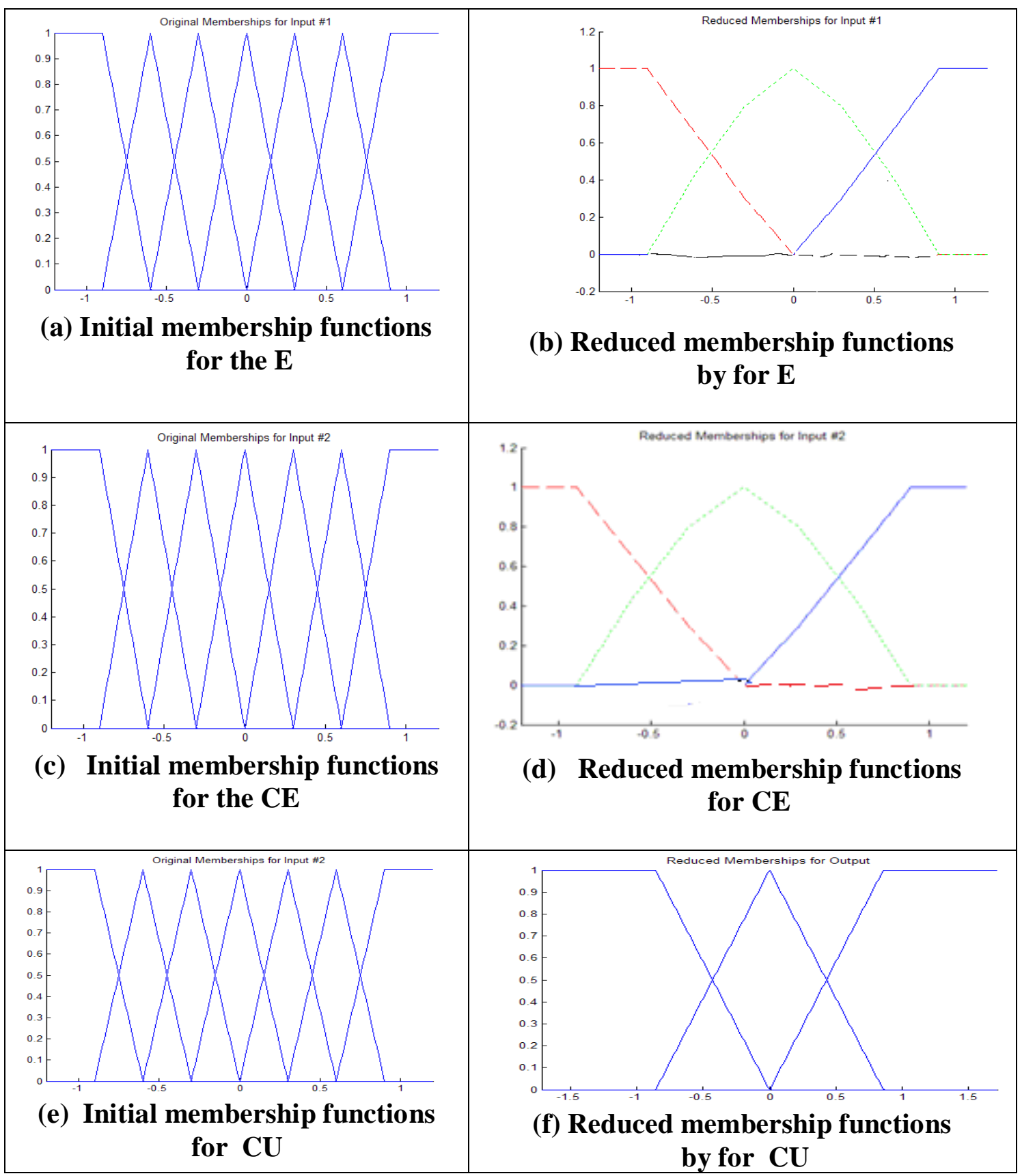

Fig. 4 Initial and modified membership functions (using SVD method) for the inputs and the output

From Table (2) we conclude that:

- The initial FLC has less overshot than that in the reduced FLC by SVD.

- The reduced FLC by SVD has faster rise time than that in the initial FLC.

- The initial FLC has less steady state error than that in the reduced FLC by SVD .SVD makes the system at sustained oscillation mode.

In order to improve the performance of the FLC, the rules and membership functions are adjusted by making the area of membership functions near $\mathrm{ZE}$ region narrower to produce finer control resolution. On the other hands, making the area far from ZE region wider gives faster control response. Also, the performance can be improved by changing the severity of the rules after adjusting the rules and membership functions, the final rules and membership functions are obtained as shown in, Table (3) and figure (6) respectively. 
Observing the performance of the system after applying the modified rules we get that there are some unused rules and in order to reduce memory size and have a faster performance we may eliminate the unused rules, so we get only 33 rules, table (4) shows the reduced modified rules.

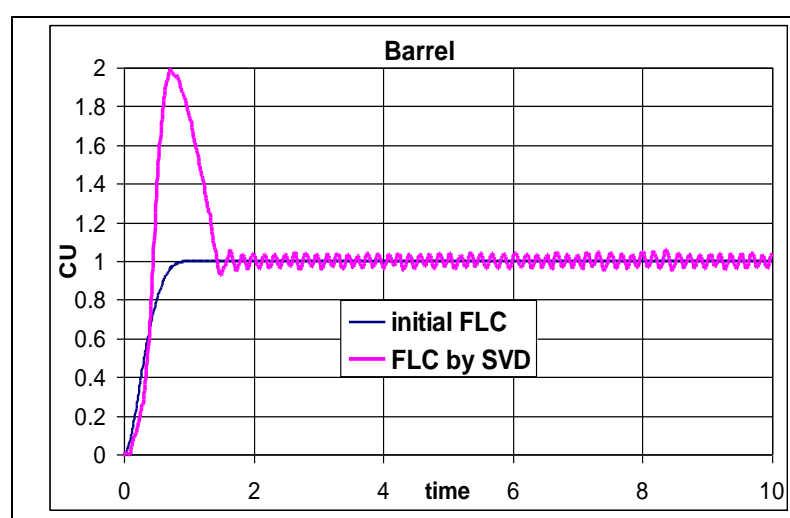

(a) Step responses of the Barrel

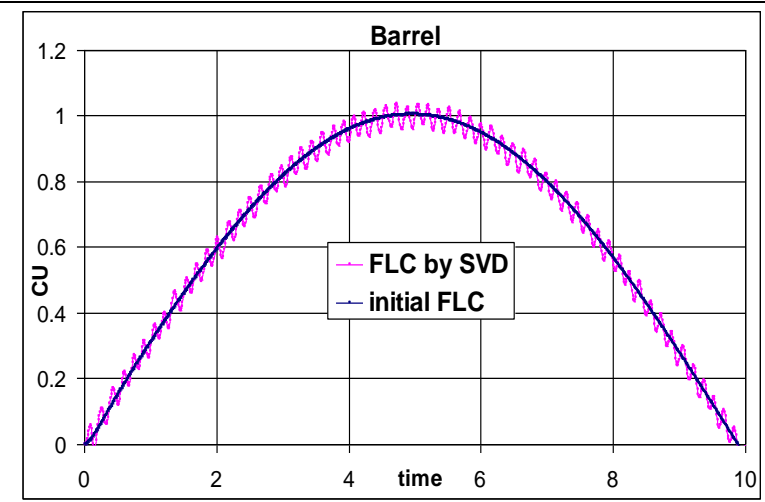

(c) Sinusoidal responses of the Barrel

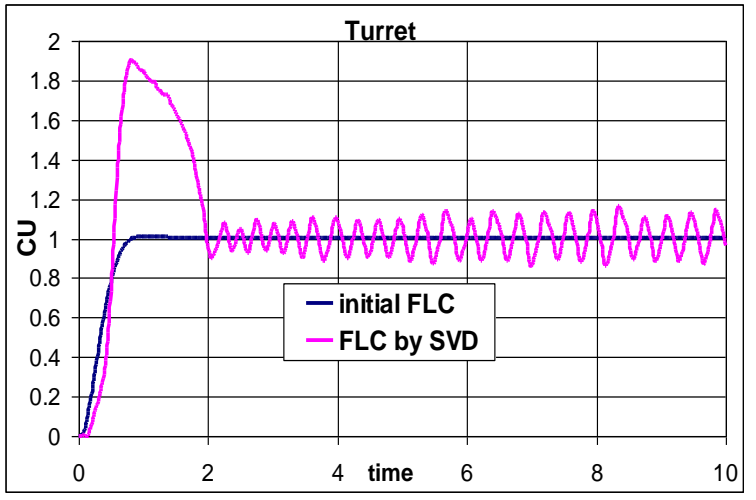

(b) Step responses of the Turret

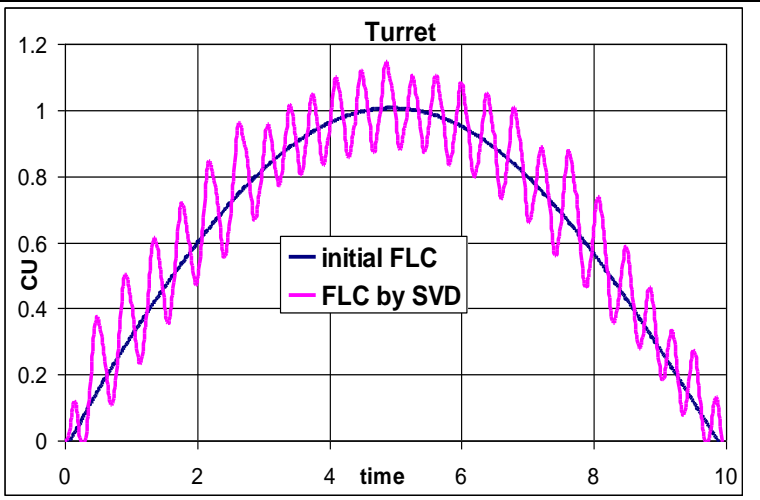

(d) Sinusoidal responses of the Turret

Fig. 5 Step and Sinus System's responses under different FLC controllers

Table 2 Comparison between initial and FLC and SVDFLC

\begin{tabular}{|c|c|c|c|c|}
\hline \multirow{2}{*}{} & \multicolumn{2}{|c|}{ Turret } & \multicolumn{2}{c|}{ Barrel } \\
\cline { 2 - 5 } & $\begin{array}{c}\text { Initial } \\
\text { FLC }\end{array}$ & SVD FLC & $\begin{array}{c}\text { Initial } \\
\text { FLC }\end{array}$ & SVD FLC \\
\hline $\begin{array}{c}\text { Steady } \\
\text { state error }\end{array}$ & 0 & $\begin{array}{c}\text { 0.23 Sustained } \\
\text { oscillation }\end{array}$ & 0 & $\begin{array}{c}0.05 \\
\text { Sustained oscillation }\end{array}$ \\
\hline overshot & 0 & 0.9 & 0 & 0.9 \\
\hline rise time & 1.06 & 0.3 & 1.01 & 0.26 \\
\hline $\begin{array}{c}\mathbf{1 0 0 1} \\
\sum\end{array}$ & 55.760 & 104.689 & 54.779 & 75.3147 \\
\hline $\mathbf{1}$ & & & & \\
\hline
\end{tabular}


Table 3 Modified rules

\begin{tabular}{|c|c|c|c|c|c|c|c|}
\hline ee & NB & NM & NS & ZE & PS & PM & PB \\
\hline NB & NB & NB & NB & NB & ZE & PM & PM \\
\hline NM & NB & NB & NB & NM & PS & PM & PB \\
\hline NS & NB & NB & NM & NS & PM & PM & PB \\
\hline ZE & NB & NM & NS & ZE & PB & PB & PB \\
\hline PS & NM & NS & ZE & PS & PB & PB & PB \\
\hline PM & NS & ZE & PS & PM & PB & PB & PB \\
\hline PB & ZE & PS & PM & PB & PB & PB & PB \\
\hline
\end{tabular}

Table 4 Reduced modified rules

\begin{tabular}{|c|c|c|c|c|c|}
\hline ce & NS & ZE & PS & PM & PB \\
\hline NB & -- & NB & ZE & PM & PM \\
\hline NM & -- & NM & PS & PM & PB \\
\hline NS & NM & NS & PM & PM & PB \\
\hline ZE & NS & ZE & PB & PB & PB \\
\hline PS & ZE & PS & PB & PB & PB \\
\hline PM & PS & PM & PB & PB & PB \\
\hline PB & PM & PB & PB & PB & PB \\
\hline
\end{tabular}

\section{Performance Evaluation}

An initial 49 rules FLC is used to control the system, regarding the performance of the system under the control of this initial 49-rules FLC, modifications in the membership functions and rules are done to have a better performance for the system. Although, we noticed the existence of some rules which are not used, so a reduction of the number of rules has been done.

Figure (7) shows the performance of the system under the control of each of a 49 rules FLC, modified (rules and membership functions) FLC, reduced modified rules FLC, it is clear that the modification in the rule base and membership functions enhances the performance of the system and from simulation results we can have the following conclusions: Rising times and overshot according to the modified FLC are better than in the original one, the modified FLC responds with less overshot and minimum settling time, the modified controller showed a good position tracking performance, and the modified controller uses less memory size.

Comparison between the system performances under the control of each of the initial 49-FLC, modified FLC, and reduced modified FLC is summarized in table (5):

- The reduced modified FLC has faster rise time than that in the initial 49 rules FLC.

- The reduced modified FLC utilizes less memory than the initial FLC does. 

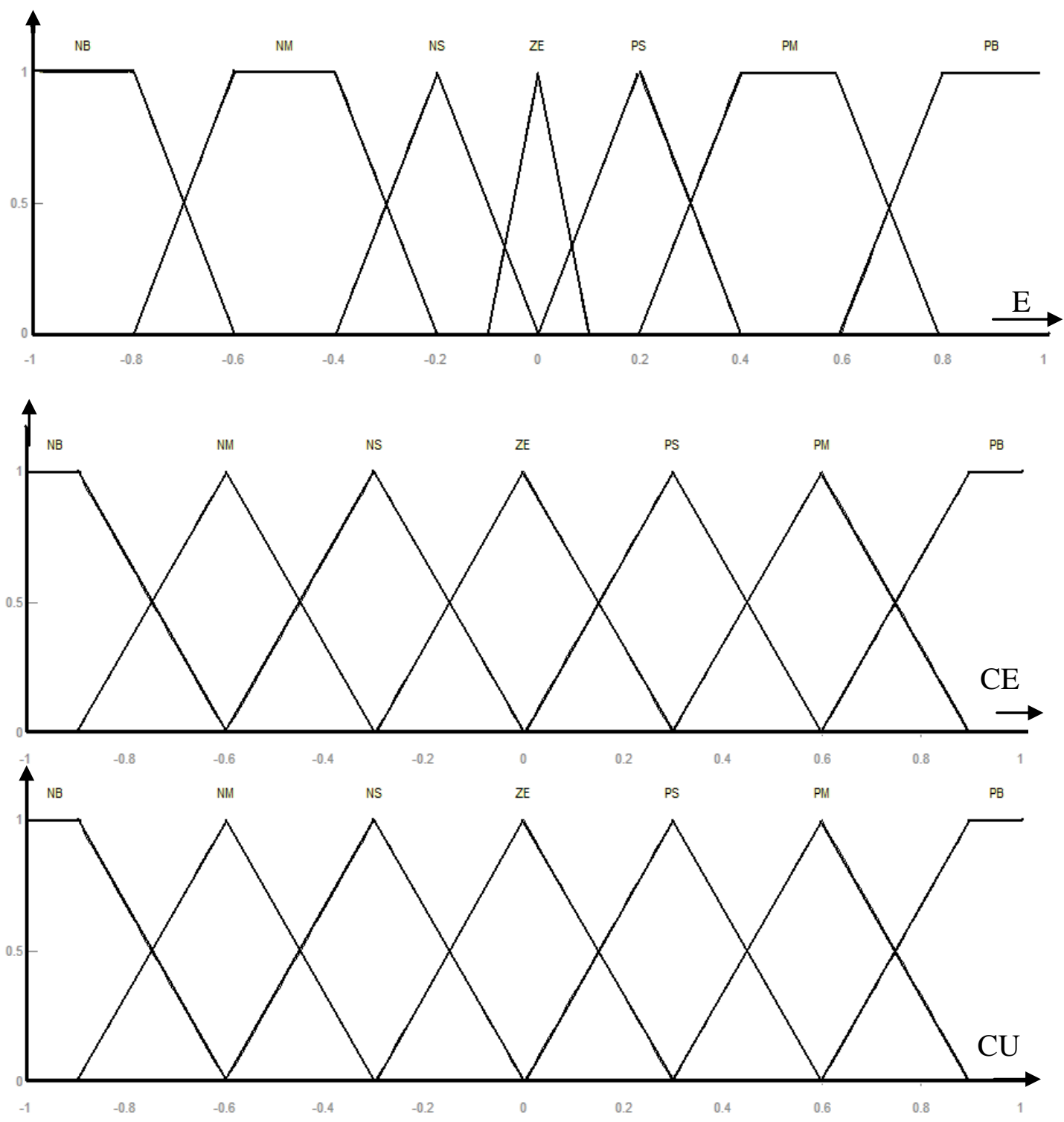

Fig. 6 Modified membership function

Table 5 Comparison between the system performance

\begin{tabular}{|c|c|c|c|c|c|c|}
\hline & \multicolumn{3}{|c|}{ Turret } & \multicolumn{3}{c|}{ Barrel } \\
\cline { 2 - 7 } & $\begin{array}{c}\text { Initial } \\
\text { 49-FLC }\end{array}$ & $\begin{array}{c}\text { Modified } \\
\text { FLC }\end{array}$ & $\begin{array}{c}\text { Reduced } \\
\text { modified } \\
\text { FLC }\end{array}$ & $\begin{array}{c}\text { Initial } \\
\text { 49-FLC }\end{array}$ & $\begin{array}{c}\text { Modified } \\
\text { FLC }\end{array}$ & $\begin{array}{c}\text { Reduced } \\
\text { modified } \\
\text { FLC }\end{array}$ \\
\hline $\begin{array}{c}\text { Steady state } \\
\text { error }\end{array}$ & 0 & 0 & 0 & 0 & 0 & 0 \\
\hline overshot & 0 & 0 & 0 & 0 & 0 & 0 \\
\hline rise time & 1.94 & 1.01 & 1.01 & 1.99 & 1.01 & 1.01 \\
\hline $\begin{array}{c}\mathbf{1 0 0 1} \\
\mathbf{1}_{\mathbf{1}}|\mathrm{e}|^{\mathbf{2}}\end{array}$ & 55.760 & 40.523 & 40.523 & 54.779 & 39.121 & 39.121 \\
\hline
\end{tabular}




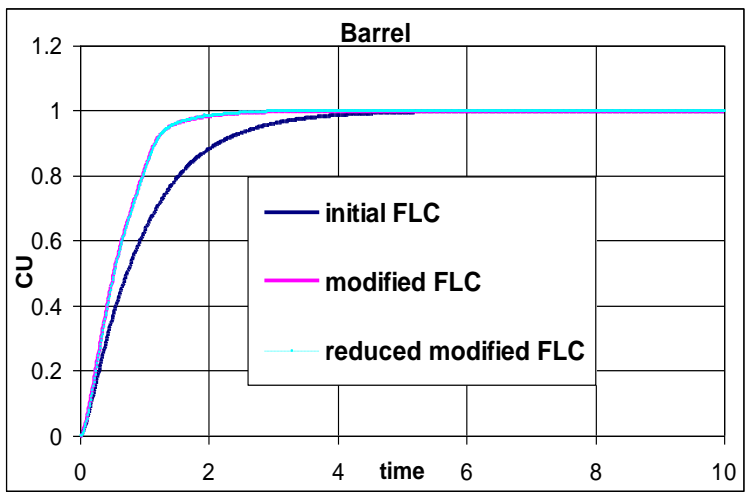

(a) Step responses of the Barrel under different controllers

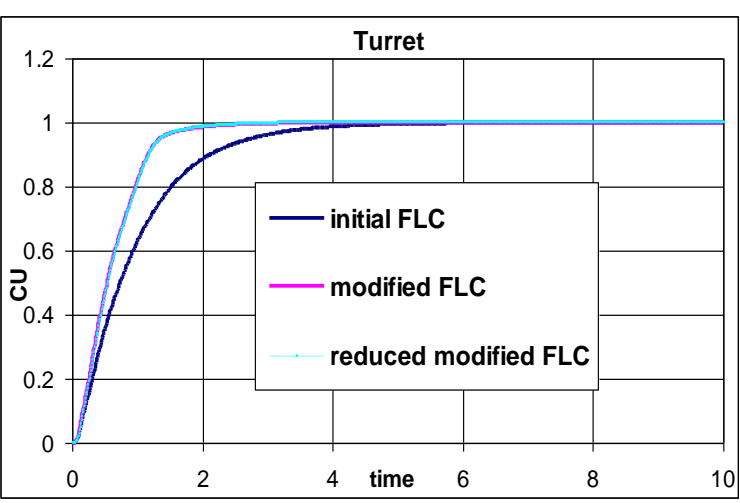

(b) Step responses of the Turret under different controllers

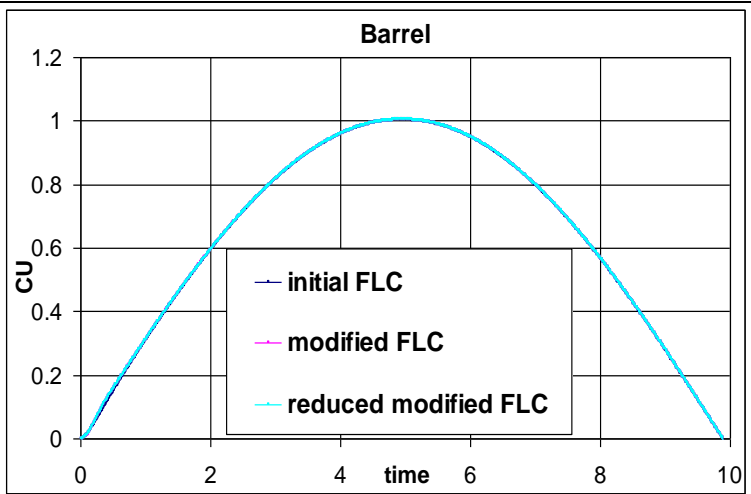

(c) Sinusoidal responses of the Barrel under different controllers

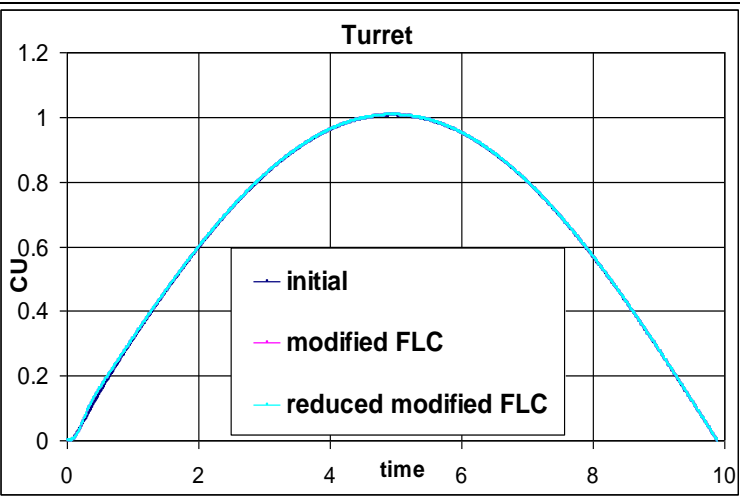

(d) Sinusoidal responses of the Turret under different controllers

Fig. 7 Step and Sinus System's responses under different FLC controllers

\section{Conclusion}

The mathematical model of a gun-turret system was derived by using (DH) convention, Lagrange's Equations of Motion is used to get each of the Turret's equation, and the Barrel's equation of motion. The derived system is a multi-input multi-output, coupled, and nonlinear system. Matlab/Simulink software is used to simulate the mathematical model of the Gun Turret-Barrel system derived by two motors. Different controllers are studied and then applied to the mathematical model of the system. A comparison between the system performances is considered under the control of each of FLC, a reduced FLC (in the number of membership functions and rules by using SVD method), and a reduced modified FLC (reduction in the number of rules and modification in the shape of the membership functions by observing the system performance). From comparisons, results may be summarized as follows: The reduced FLC by SVD method utilizes less memory, but it has unacceptable response for the gun turret-barrel system. The reduced modified FLC by observing the system performance performs better than the reduced one by using SVD method, but it utilizes more memory. The reduced modified FLC has less overshot, faster rise time, and less steady state error than that in the initial FLC. 


\section{References}

[1] Fire control systems - general, MIL-HDBK-799 (AR), April 1996

[2] Daniel J. Pack, "Comparative Study of Motion Control Methods: A Case Study for a Non Linear System", Journal of the Society for Computer Simulation, Vol. 67, No. 1, July 1996, pp. 35-49.

[3] E.M. Ramzy, "Robotic Manipulator Arm Control Using the Adaptive Gain Scheduling PID Controller7', M.Sc. Thesis, Cairo University, Cairo, 1996.

[4] Jose Ragot and Michel Lamotte, "Fuzzy Logic Control", Inter. Jour. System Sci, Vol. 24, No. 10, 1993, pp. 1825-1848.

[5] M. Mizumoto, "Fuzzy controls by product-sum-gravity method", advancement of Fuzzy Theory and Systems in China and Japan, Eds. Liu and Mizumoto, International Academic Publishers, c1.1.-c.1.4. 1990.

[6] C.T.Yang, P.Baranyi, Y.Yam and Sz.Kovács, "SVD Reduction of a Fuzzy Controller in an AGV Steering System” EFDAN'99, Dortmund, Germany, 1999, pp 118-124.

[7] P.Baranyi and Y.Yam, "Singular Value-Based Approximation with Non- Singleton Fuzzy Rule Base", 7th Int. Fuzzy Systems Association World Congress (IFSA'97), Prague, 1997, pp. 127-132.

[8] Yeung Yam, Péter Baranyi and Chi Tin Yang, "Reduction of Fuzzy Rule Base Via Singular Value Decomposition" IEEE Trans. on Fuzzy Systems. Vol.: 7, No. 2, ISSN 1063-6706, 1999, pp. 120-131.

[9] P.Baranyi, Y.Yam and Chi-Tin Yang, "Singular Value Decomposition of Linguistic Symbol-Array", IEEE Conference on Systems Man and Cybernetics (IEEE SMC'99), 1999, Tokyo, Japan, pp.:III/822-III/826.

[10] Péter Baranyi and Yeung Yam "Fuzzy rule base reduction", Theory and Applications Eds., D. Ruan and E.E. Kerre, Kluwer, 2000, pp 135-160. 